\title{
Multivariate winning probabilities
}

\author{
Ignacio Montes \\ UNIMODE, Department of Statistics and O.R., University of Oviedo, C/Federico García \\ Lorca 18, 33007 Oviedo, Spain. E-mail: imontes@uniovi.es \\ Susana Montes \\ UNIMODE, Department of Statistics and O.R., University of Oviedo, C/Federico García \\ Lorca 18,33007 Oviedo, Spain. E-mail: montes@uniovi.es \\ Bernard De Baets \\ KERMIT, Department of Data Analysis and Mathematical Modelling, Ghent University, \\ Coupure links 653, 9000 Ghent, Belgium. E-mail: Bernard.DeBaets@UGent.be
}

\begin{abstract}
Stochastic dominance and statistical preference are two important tools for the pairwise comparison of random variables. However, pairwise methods are not always appropriate in the case of more than two alternatives. In this work, we generalize the notion of winning probability to the notion of multivariate winning probability. The latter allows to establish a ranking (with ties) on any set of random variables and naturally leads to the notion of probabilistic preference. We investigate the relationship between the latter notion and the classical notions of stochastic dominance and statistical preference.

Keywords: Winning probabilities, Stochastic dominance, Probabilistic relation, Statistical preference, Decision Making

\section{Introduction}

In decision making under risk, the different alternatives under consideration are usually modelled by means of random variables, whence the need arises for tools that allow to establish an ordering between random variables. Two of the most prominent such tools are stochastic dominance [1, 2, 3] and statistical preference [4, 5].
\end{abstract}

Preprint submitted to Fuzzy Sets and Systems

September 22, 2018 
Stochastic dominance is probably the most widely used tool for ordering random variables. Over the last decades it has been applied in a variety of areas, such as economics [1, agriculture [6], operations research [7, and so on. However, stochastic dominance does not take into account the possible dependence between the random variables under consideration, since it is based on their marginal distributions only. To cope with this problem, various generalizations have been proposed, such as the stochastic precedence order [8, 9] and probability dominance 10. From the same point of view, statistical preference has been introduced [4, 5] as a graded version of stochastic dominance, in the sense that it is based on a probabilistic relation that expresses the winning probabilities between the random variables.

Stochastic dominance between two random variables can be characterized in terms of the comparison of the expectations of increasing transformations of these random variables. In this vein, some of the present authors have proved that statistical preference is closer to another location parameter, namely the median [1]. We have also proved that, under common conditions such as independence, (first degree) stochastic dominance implies statistical preference [12, 13, 14, 15, and that both concepts are equivalent in some particular situations, such as when comparing normally distributed random variables with the same variance [16].

In this work, we will focus on the problem of comparing more than two random variables. In this setting, pairwise methods like stochastic dominance and statistical preference have a number of drawbacks, whence the need arises to develop alternative comparison tools. To that end, we will generalize the notion of winning probability for a set of random variables. These so-called multivariate winning probabilities are computed using the joint distribution of all the random variables involved and thus use all the available information. The multivariate winning probabilities allow to rank (with ties) the random variables, and thus naturally lead to a preference relation called probabilistic preference, which is an extension of the notion of statistical preference to a multivariate setting. In addition, we will characterize probabilistic preference in terms of 
the comparison of certain cumulative distribution functions, and we will investigate its relationship with statistical preference. We will also investigate the relationship between probabilistic preference and stochastic dominance in the case of independent random variables. Finally, we will illustrate how to use the multivariate winning probabilities and the associated probabilistic preference relation in a linguistic decision making problem.

This contribution is organized as follows. In Section 2, we will give an overview of the notions of stochastic dominance and statistical preference, as well as other stochastic orders like expected utility [17] and multi-utility representations [18]. We will recall a number of characterizations and the connections between them. In Section 3, we will discuss why these pairwise methods are not fully adequate for comparing more than two random variables and we will introduce multivariate winning probabilities, which will give rise to the notion of probabilistic preference. In Section 4 we will investigate some of the properties of this new tool, while in Section 5 we will investigate the relationship between (first degree) stochastic dominance and probabilistic preference in the case of independent random variables. Finally, we will demonstrate in Section 6 how probabilistic preference can be applied in a decision making problem and we will devote the final section to conclude the paper and to discuss possible future lines of research.

\section{Pairwise comparison of random variables}

Throughout this paper, we consider a probability space $(\Omega, \Sigma, P)$. A random variable $X: \Omega \rightarrow \mathbb{R}$ is a $\Sigma$-measurable function. Associated with the random variable $X$, we consider its cumulative distribution function $F_{X}: \mathbb{R} \rightarrow[0,1]$, given by $F_{X}(t)=P(X \leq t)$, where $\{X \leq t\}$ denotes the set $\{\omega \in \Omega \mid X(\omega) \leq t\}$.

The pairwise comparison of random variables is a topic that has been widely studied in the literature, and several methods have been proposed over the last decades. In this section, we recall the notions of stochastic dominance and statistical preference, and explain the relationship between them. 
The best-known stochastic order is the expected utility model of von Neumann and Morgenstern [17]: if $u: \mathbb{R} \rightarrow \mathbb{R}$ is a utility function, then a random variable $X$ is preferred to a random variable $Y$, denoted by $X \succeq_{\mathrm{EU}} Y$, if $E[u(X)] \geq E[u(Y)]$, assuming that both expectations exist.

A more general approach was proposed in [18]: if $\mathcal{U}$ denotes a set of utility functions, then $X$ is preferred to $Y$ with respect to the multi-utility representation $\mathcal{U}$, denoted by $X \succeq_{\mathrm{MUR}} Y$, if $E[u(X)] \geq E[u(Y)]$ for every $u \in \mathcal{U}$.

Another possibility is to use stochastic dominance, which is based on the direct comparison of the cumulative distribution functions associated with the random variables $[1,2,3$.

Definition 1. Let $X$ and $Y$ be two random variables with cumulative distribution functions $F_{X}$ and $F_{Y} . X$ is said to stochastically dominate $Y$ in the first degree, denoted by $X \succeq_{\mathrm{FSD}} Y$, if $F_{X}(t) \leq F_{Y}(t)$ for any $t \in \mathbb{R}$.

Stochastic dominance between two random variables can be characterized in terms of the comparison of the expectations of some appropriate transformations of these random variables (see, for example, [1]):

$$
X \succeq_{\mathrm{FSD}} Y \quad \Longleftrightarrow \quad E[u(X)] \geq E[u(Y)]
$$

for any increasing function $u$, whenever both expectations exist. We can easily see that stochastic dominance is equivalent to the multi-utility representation considering for $\mathcal{U}$ the set of all increasing utility functions. For an extensive treatment of the notion of stochastic dominance, we refer to [1, 2, 3].

The notion of statistical preference is related to that of a probabilistic relation.

Definition 2. Given a set $\mathcal{A}$, a mapping $Q: \mathcal{A} \times \mathcal{A} \rightarrow[0,1]$ is called a probabilistic relation on $\mathcal{A}$ if it fulfills the reciprocity property:

$$
Q(a, b)+Q(b, a)=1
$$

for any $(a, b) \in \mathcal{A} \times \mathcal{A}$. 
In [4, 5], $\mathcal{A}$ is formed by random variables defined on the same probability space, and the following probabilistic relation is defined.

Definition $3([4,5])$. Let $\mathcal{A}$ denote a set of random variables defined on the probability space $(\Omega, \Sigma, P)$. The winning probability relation on $\mathcal{A}$ is the probabilistic relation $Q$ on $\mathcal{A}$ defined by

$$
Q(X, Y)=P(X>Y)+\frac{1}{2} P(X=Y) .
$$

The above probabilistic relation expresses the winning probability of a random variable $X$ over another random variable $Y$, in the sense that the greater the winning probability $Q(X, Y)$, the stronger the preference of $X$ over $Y$. Hence, the closer the value $Q(X, Y)$ to 1 , the more $X$ is preferred over $Y$; the closer $Q(X, Y)$ to 0 , the more $Y$ is preferred over $X$; and if $Q(X, Y)$ equals 0.5 , then both random variables are considered indifferent.

Statistical preference is the formal interpretation of the winning probability relation $Q$.

Definition $4([4,5])$. Let $X$ and $Y$ be two random variables defined on the same probability space:

(i) $X$ is said to be statistically preferred to $Y$, denoted by $X \succeq_{\mathrm{SP}} Y$, if $Q(X, Y) \geq \frac{1}{2}$

(ii) $X$ is said to be strictly statistically preferred to $Y$, denoted by $X \succ_{\mathrm{SP}} Y$, if $Q(X, Y)>\frac{1}{2}$;

(iii) $X$ and $Y$ are said to be statistically indifferent if $Q(X, Y)=\frac{1}{2}$.

One obvious advantage of statistical preference compared to stochastic dominance is the possibility of establishing winning probabilities between the alternatives. Another advantage is the fact that it takes into account the possible dependence between the random variables since it is based on the joint distribution, while stochastic dominance only uses the marginal distributions. Moreover, statistical preference establishes a complete relation, while we can find 
pairs of random variables that are incomparable under first degree stochastic dominance.

Taking into account that $Q(X, Y) \geq Q(Y, X)$ if and only if $P(X \geq Y) \geq$ $P(Y \geq X)$, statistical preference can be characterized in the following way.

Theorem 5 ([1] ). Let $X$ and $Y$ be two random variables defined on the same probability space with cumulative distribution functions $F_{X}$ and $F_{Y}$. The following equivalence holds:

$$
X \succeq_{\mathrm{SP}} Y \quad \Longleftrightarrow \quad F_{X-Y}(0) \leq F_{Y-X}(0) .
$$

\section{General comparison of random variables using multivariate win- ning probabilities}

This section introduces a generalization of the notion of statistical preference for the comparison of more than two random variables. First, we explain why the pairwise methods discussed above may not be adequate in this setting, and then we introduce the notion of multivariate winning probability, which gives rise to a ranking (with ties) on any set of random variables.

\subsection{Pairwise methods for comparing more than two random variables}

So far, we have presented stochastic dominance and statistical preference as pairwise comparison methods. However, a natural question arises: can we use these methods for the comparison of more than two random variables? On the one hand, stochastic dominance was defined as a pairwise comparison method based on the pointwise comparison of cumulative distribution functions. This method could be adapted for comparing more than two random variables, saying that a random variable is the preferred one if it stochastically dominates all the other ones. However, as we have already mentioned, stochastic dominance allows for incomparability, and, moreover, different random variables could have the same cumulative distribution function. Thus, stochastic dominance results in a pre-order (also called quasi-order) only. 
While statistical preference does not allow for incomparability, it has its own important drawback: its lack of transitivity in general. The underlying idea of statistical preference is to consider $X$ preferred to $Y$ when it provides a greater utility the majority of times. As such, it is close to the rule of majority in voting systems; taking into account Condorcet's paradox [19, it is not difficult to see that statistical preference is not always transitive. De Schuymer et al. [4, 5] provided an example to illustrate this fact; another one can be found in 20. Example 3].

Example 6 ([5, Section 1]). Consider three fair dice. Each dice has the following values on its faces:

\begin{tabular}{ll|cccccc} 
& Notation & face 1 & face 2 & face 3 & face 4 & face 5 & face 6 \\
\hline First dice & $X$ & 1 & 3 & 4 & 15 & 16 & 17 \\
Second dice & $Y$ & 2 & 10 & 11 & 12 & 13 & 14 \\
Third dice & $Z$ & 5 & 6 & 7 & 8 & 9 & 18
\end{tabular}

We can then consider the probability space $(\Omega, \mathcal{P}(\Omega), P)$, where the elements $\omega \in \Omega$ are given by

$\omega=$ (Face $i$ of the first dice, Face $j$ of the second dice, Face $k$ of the third dice)

for any $i, j, k \in\{1, \ldots, 6\}$. Since we are assuming that the dice are fair, $P$ is the discrete uniform distribution.

We consider the game consisting of rolling the three dice simultaneously, so that the dice whose number is the greatest wins the game. We compute the winning probability relation $Q$ for these dice, and obtain:

$$
\begin{aligned}
& Q(X, Y)=\frac{5}{9}, \text { i.e. } X \succ_{\mathrm{SP}} Y, \\
& Q(Y, Z)=\frac{25}{36}, \text { i.e. } Y \succ_{\mathrm{SP}} Z, \\
& Q(Z, X)=\frac{7}{12}, \text { i.e. } Z \succ_{\mathrm{SP}} X .
\end{aligned}
$$

Hence, dice $X$ is strictly statistically preferred to dice $Y$, dice $Y$ is strictly statistically preferred to dice $Z$, yet dice $Z$ is strictly statistically preferred to dice $X$, i.e., there is a cycle. 
This fact has already been profoundly studied by some authors, like De Baets, De Meyer, De Loof and De Schuymer [5, 15, 21, 22, 23, 24, 25, 26, 27] and Martinetti et al. 28. The above example shows that statistical preference may not be adequate when we want to compare more than two random variables, precisely because it is based on pairwise comparisons only.

Since there are situations in which both stochastic dominance and statistical preference may not be adequate for the comparison of more than two random variables, our aim in this section is to propose a generalization of the notion of statistical preference suitable for the comparison of $n$ random variables, based on a generalization of the winning probability relation defined in Eq. 22). After introducing the main definition, in Section 4 we will investigate its properties, possible characterizations and the relationship with statistical preference.

\subsection{Multivariate winning probabilities}

First of all, we are going to analyze the case of three random variables, as in the dice example, and later we will generalize our definition to the case of $n$ random variables.

Let us consider a set $\mathcal{A}=\{X, Y, Z\}$ formed by three distinct random variables $X, Y$ and $Z$ defined on the probability space $(\Omega, \Sigma, P)$. We can decompose $\Omega$ in the following way:

$$
\begin{aligned}
\Omega= & \{X>\max (Y, Z)\} \cup\{Y>\max (X, Z)\} \cup\{Z>\max (X, Y)\} \\
& \cup\{X=Y>Z\} \cup\{X=Z>Y\} \cup\{Y=Z>X\} \cup\{X=Y=Z\} .
\end{aligned}
$$

Obviously, $\{X>\max (Y, Z)\}$ denotes the subset of $\Omega$ containing the elements $\omega \in \Omega$ satisfying $X(\omega)>\max (Y(\omega), Z(\omega))$, and similarly for the other sets. In the remainder, we will use this shorthand notation for the sake of brevity.

The above decomposition yields a partition of $\Omega$. As a consequence,

$$
\begin{aligned}
1= & P(X>\max (Y, Z))+P(Y>\max (X, Z))+P(Z>\max (X, Y)) \\
& +P(X=Y>Z)+P(X=Z>Y)+P(Y=Z>X)+P(X=Y=Z) .
\end{aligned}
$$


Our goal now is to define the multivariate winning probability of $X$ in $\mathcal{A}=$ $\{X, Y, Z\}$. For this aim, we define $\Pi_{\mathcal{A}}(X)$ as follows:

$$
\begin{aligned}
\Pi_{\mathcal{A}}(X)= & P(X>\max (Y, Z)) \\
& +\frac{1}{2}(P(X=Y>Z)+P(X=Z>Y))+\frac{1}{3} P(X=Y=Z) .
\end{aligned}
$$

This expression generalizes Eq. (2), and therefore we will refer to $\Pi_{\mathcal{A}}(X)$ as the multivariate winning probability of $X$ in $\mathcal{A}$. Furthermore, we can also consider the multivariate winning probabilities of $Y$ and $Z$ in $\mathcal{A}$, denoted by $\Pi_{\mathcal{A}}(Y)$ and $\Pi_{\mathcal{A}}(Z)$, given by:

$$
\begin{aligned}
\Pi_{\mathcal{A}}(Y)= & P(Y>\max (X, Z))+\frac{1}{2}(P(X=Y>Z)+P(Y=Z>X)) \\
& +\frac{1}{3} P(X=Y=Z), \\
\Pi_{\mathcal{A}}(Z)= & P(Z>\max (X, Y))+\frac{1}{2}(P(X=Z>Y)+P(Y=Z>X)) \\
& +\frac{1}{3} P(X=Y=Z) .
\end{aligned}
$$

Using the partition of $\Omega$ introduced in Eqs. (3) and (4), it can be shown that:

$$
\Pi_{\mathcal{A}}(X)+\Pi_{\mathcal{A}}(Y)+\Pi_{\mathcal{A}}(Z)=1 .
$$

In this sense, following the idea of De Schuymer et al. [4, 5], $X$ is the preferred random variable in $\mathcal{A}$, with multivariate winning probability $\Pi_{\mathcal{A}}(X)$, if

$$
\Pi_{\mathcal{A}}(X) \geq \max \left(\Pi_{\mathcal{A}}(Y), \Pi_{\mathcal{A}}(Z)\right) .
$$

Let us now extend this idea to a more general setting. Let $\mathcal{A}$ denote a finite set of distinct random variables defined on the same probability space $(\Omega, \Sigma, P)$. Then, we can consider the mapping:

$$
\Pi_{\mathcal{A}}: \mathcal{A} \rightarrow[0,1]
$$

defined for any $X \in \mathcal{A}$ by

$$
\Pi_{\mathcal{A}}(X)=\sum_{\mathcal{Y} \subseteq \mathcal{A} \backslash\{X\}} \frac{1}{1+|\mathcal{Y}|} P((\forall Z \in \mathcal{Y})(\forall W \in \mathcal{A} \backslash(\{X\} \cup \mathcal{Y}))(X=Z>W)) .
$$

\footnotetext{
${ }^{1}$ Note again that the notation $\{(\forall Z \in \mathcal{Y})(\forall W \in \mathcal{A} \backslash(\{X\} \cup \mathcal{Y}))(X=Z>W)\}$ in Eq. (5) is a shorthand for $\{\omega \in \Omega \mid(\forall Z \in \mathcal{Y})(\forall W \in \mathcal{A} \backslash(\{X\} \cup \mathcal{Y}))(X(\omega)=Z(\omega)>W(\omega))\}$.
} 
Note that this formula is a generalization of the probabilistic relation defined in Eq. (2), since for $\mathcal{A}=\{X, Y\}$ we retrieve Eq. 22). We can interpret the value of $\Pi_{\mathcal{A}}(X)$ as the multivariate winning probability of $X$ in the set of random variables $\mathcal{A}$. Consequently, the greater the multivariate winning probability $\Pi_{\mathcal{A}}(X)$, the stronger the preference for $X$ in $\mathcal{A}$. Since any random variable in $\mathcal{A}$ has an associated multivariate winning probability, we can establish a ranking (with ties) on $\mathcal{A}$. If $\Pi_{\mathcal{A}}\left(X_{\sigma(1)}\right) \geq \Pi_{\mathcal{A}}\left(X_{\sigma(2)}\right) \geq \ldots \geq \Pi_{\mathcal{A}}\left(X_{\sigma(|\mathcal{A}|)}\right)$, then $X_{\sigma(1)}$ would be the preferred random variable, $X_{\sigma(2)}$ would be the second preferred random variable and so on. This ranking gives rise to the notion of probabilistic preferenct ${ }^{2}$,

Definition 7. Let $\mathcal{A}$ be a finite set of distinct random variables. Given $X, Y \in$ $\mathcal{A}$ :

(i) $X$ is said to be probabilistically preferred to $Y$ in $\mathcal{A}$, denoted by $X \succeq_{\mathrm{PP}}^{\mathcal{A}} Y$, if $\Pi_{\mathcal{A}}(X) \geq \Pi_{\mathcal{A}}(Y)$

(ii) $X$ is said to be strictly probabilistically preferred to $Y$ in $\mathcal{A}$, denoted by $X \succ_{\mathrm{PP}}^{\mathcal{A}} Y$, if $\Pi_{\mathcal{A}}(X)>\Pi_{\mathcal{A}}(Y) ;$

(iii) $X$ is said to be probabilistically indifferent to $Y$ in $\mathcal{A}$, denoted by $X \equiv_{\mathrm{PP}}^{\mathcal{A}} Y$, if $\Pi_{\mathcal{A}}(X)=\Pi_{\mathcal{A}}(Y)$.

Similarly, given $X \in \mathcal{A}, X$ is probabilistically preferred to the random variables in $\mathcal{A} \backslash\{X\}$, denoted by $X \succeq_{\mathrm{PP}}^{\mathcal{A}} \mathcal{A} \backslash\{X\}$, if $\Pi_{\mathcal{A}}(X) \geq \Pi_{\mathcal{A}}(Y)$ for any $Y \in \mathcal{A} \backslash\{X\}$.

For the sake of simplicity, whenever no confusion is possible, we will remove the superscript in $\succeq_{\mathrm{PP}}^{\mathcal{A}}, \succ_{\mathrm{PP}}^{\mathcal{A}}$ and $\equiv_{\mathrm{PP}}^{\mathcal{A}}$.

Note that probabilistic preference is transitive, in contrast with statistical preference, because it is based on the comparison of the multivariate winning probabilities, so $\succeq_{\mathrm{PP}}$ is a weak ordering allowing for ties.

\footnotetext{
${ }^{2}$ We prefer the term probabilistic preference instead of statistical preference, because it emphasizes that we are dealing with probability distributions rather than with statistical data.
} 
As was the case for statistical preference, probabilistic preference is based on the multivariate winning probabilities, hence it is based on the joint distribution of the variables in $\mathcal{A}$, and thus takes into account the stochastic dependences between them. Furthermore, the mapping $\Pi_{\mathcal{A}}$ also satisfies the property:

$$
\sum_{X \in \mathcal{A}} \Pi_{\mathcal{A}}(X)=1
$$

This means that $\Pi_{\mathcal{A}}$ can be seen as a probability distribution on $\mathcal{A}$, which measures the multivariate winning probability of each random variable in $\mathcal{A}$. Also, if we know $|\mathcal{A}|-1$ of the multivariate winning probabilities, the remaining one directly follows because their sum equals 1 . Moreover, when one of the multivariate winning probabilities takes a value larger than 0.5 , the corresponding random variable will surely be the most preferred one.

Example 8. We consider again the framework in Example 6 and compute the multivariate winning probabilities to find the preferred dice in the set $\mathcal{A}=$ $\{X, Y, Z\}: \Pi_{\mathcal{A}}(X)=0.4167 ; \Pi_{\mathcal{A}}(Y)=0.3472$; and $\Pi_{\mathcal{A}}(Z)=0.2361$. Thus, $X$ is the most preferred dice with multivariate winning probability 0.4167; $Y$ is the second most preferred dice with multivariate winning probability 0.3472; and $Z$ is the least preferred dice with multivariate winning probability 0.2361.

\section{Properties of probabilistic preference}

In this section we investigate some basic properties of probabilistic preference. First, we study the relationship between (pairwise) statistical preference and probabilistic preference. Second, we generalize Theorem 5 providing a characterization of probabilistic preference.

First, we prove that probabilistic preference sometimes points out a different preferred random variable than statistical preference. This is because probabilistic preference uses the joint distribution of all the variables, while statistical preference only takes into account their bivariate distributions, and, consequently, it does not use all of the available information. 
Proposition 9. The following statements hold:

1. There exists a finite set $\mathcal{A}$ of distinct random variables containing a random variable $X$ such that

$$
(\forall Y \in \mathcal{A} \backslash\{X\})\left(X \succ_{\mathrm{SP}} Y\right)
$$

while

$$
(\exists Z \in \mathcal{A} \backslash\{X\})\left(Z \succ_{\mathrm{PP}} X\right) .
$$

2. There exists a finite set $\mathcal{A}$ of distinct random variables containing a random variable $X$ such that

$$
(\forall Y \in \mathcal{A} \backslash\{X\})\left(Y \succ_{\mathrm{SP}} X\right),
$$

while

$$
(\forall Y \in \mathcal{A} \backslash\{X\})\left(X \succ_{\mathrm{PP}} Y\right) .
$$

Proof. Let us consider the first statement. To provide an example, consider $n=2$ and the probability space $(\Omega, \mathcal{P}(\Omega), P)$, where $\Omega=\left\{\omega_{1}, \omega_{2}, \omega_{3}\right\}$ and $P\left(\left\{\omega_{1}\right\}\right)=0.4, P\left(\left\{\omega_{2}\right\}\right)=P\left(\omega_{3}\right)=0.3$. Let us consider the random variables $X, Y$ and $Z$ defined by:

\begin{tabular}{c|ccc} 
& $\omega_{1}$ & $\omega_{2}$ & $\omega_{3}$ \\
\hline$X$ & 2 & 1 & 1 \\
$Y$ & 0 & 2 & 1 \\
$Z$ & 2 & 0 & 0
\end{tabular}

It holds that $Q(X, Y)=0.55$ and $Q(X, Z)=0.8$, and, consequently, $X \succ_{\mathrm{SP}} Y$ and $X \succ_{\mathrm{SP}} Z$. However, for the set of random variables $\mathcal{A}=\{X, Y, Z\}$, it holds that

$$
\Pi_{\mathcal{A}}(X)=0.35, \quad \Pi_{\mathcal{A}}(Y)=0.45, \quad \Pi_{\mathcal{A}}(Z)=0.2 .
$$

Thus, $Y \succ_{\mathrm{PP}} X \succ_{\mathrm{PP}} Z$, so $X$ is not the preferred random variable in $\mathcal{A}=$ $\{X, Y, Z\}$.

Next, we consider the second statement. Consider $n=2$ and the dice $X, Y$ and $Z$, defined on the same probability space as in Example 6 , by:

$$
\begin{aligned}
& X=\{1,2,4,6,17,18\}, \quad Y=\{3,7,9,12,14,16\}, \\
& Z=\{5,8,10,11,13,15\} .
\end{aligned}
$$


It holds that $Y \succ_{\mathrm{SP}} X$ and $Z \succ_{\mathrm{SP}} X$, since $Q(X, Y)=\frac{7}{18}$ and $Q(X, Z)=\frac{13}{36}$. However, if we compute the multivariate winning probabilities in the set $\mathcal{A}=$ $\{X, Y, Z\}$, we obtain the following:

$$
\Pi_{\mathcal{A}}(X)=\frac{73}{216}, \quad \Pi_{\mathcal{A}}(Y)=\frac{72}{216}, \quad \Pi_{\mathcal{A}}(Z)=\frac{71}{216} .
$$

Consequently, $X \succ_{\mathrm{PP}} Y \succ_{\mathrm{PP}} Z$.

The second part of Proposition 9 implies that if $X \succeq_{\mathrm{PP}} \mathcal{A} \backslash\{X\}$ and we take a subset $\mathcal{A}^{\prime} \subset \mathcal{A}$ with $X \in \mathcal{A}^{\prime}$, there could be a random variable $Y \in \mathcal{A}^{\prime} \backslash\{X\}$ such that $\Pi_{\mathcal{A}^{\prime}}(Y)>\Pi_{\mathcal{A}^{\prime}}(X)$. In other words, the preference is not preserved in a smaller set of alternatives. In particular, this means that there is no general relationship between statistical preference and probabilistic preference, even for independent random variables.

The following result shows that, although pairwise and multivariate winning probabilities are in general not related, under some conditions we can reduce the set of alternatives $\mathcal{A}$ preserving the winning probabilities.

Proposition 10. Let $\mathcal{A}$ be a finite set of distinct random variables. Assume that there exists $X \in \mathcal{A}$ such that $X(\omega)<\max _{Y \in \mathcal{A} \backslash\{X\}} Y(\omega)$ for every $\omega \in \Omega$. Then, $\Pi_{\mathcal{A}}(X)=0$ and for every $Y \in \mathcal{A} \backslash\{X\}$ it holds that:

$$
\Pi_{\mathcal{A}}(Y)=\Pi_{\mathcal{A} \backslash\{X\}}(Y) .
$$

Proof. Since $X(\omega)<\max _{Y \in \mathcal{A} \backslash\{X\}} Y(\omega)$ for every $\omega \in \Omega$, it holds that:

$$
\{\omega \in \Omega \mid(\forall Y \in \mathcal{A} \backslash\{X\})(X(\omega) \geq Y(\omega))\}=\emptyset .
$$

It follows that $\Pi_{\mathcal{A}}(X)=0$.

Also, consider $Y \in \mathcal{Y}$, then for any $\mathcal{Y} \subseteq \mathcal{A} \backslash\{X, Y\}$ it holds that:

$$
\begin{aligned}
& \{\omega \in \Omega \mid(\forall Z \in \mathcal{Y})(\forall W \in \mathcal{A} \backslash(\{Y\} \cup \mathcal{Y}))(Y(\omega)=Z(\omega)>W(\omega))\}= \\
& \{\omega \in \Omega \mid(\forall Z \in \mathcal{Y})(\forall W \in \mathcal{A} \backslash(\{X, Y\} \cup \mathcal{Y}))(Y(\omega)=Z(\omega)>W(\omega))\},
\end{aligned}
$$

and therefore the multivariate winning probability $\Pi_{\mathcal{A}}(Y)$ equals $\Pi_{\mathcal{A} \backslash\{X\}}(Y)$ for every $Y \in \mathcal{A} \backslash\{X\}$. 
Next, we prove that $\Pi_{\mathcal{A}}(X)$ is always smaller than or equal to $Q(X, Y)$ for every $Y \in \mathcal{A} \backslash\{X\}$. This means that the multivariate winning probability of $X$ in $\mathcal{A}$ cannot be greater than the winning probability over any of the variables in a pairwise comparison.

Proposition 11. Let $\mathcal{A}$ be a finite set of distinct random variables. It holds that:

$$
\Pi_{\mathcal{A}}(X) \leq Q(X, Y), \text { for any } Y \in \mathcal{A} \backslash\{X\}
$$

Consequently, if $\Pi_{\mathcal{A}}(X) \geq \frac{1}{2}$, then $X \succeq_{\mathrm{PP}} \mathcal{A} \backslash\{X\}$ and $X \succeq_{\mathrm{SP}} Y$ for any $Y \in \mathcal{A} \backslash\{X\}$.

Proof. First of all, note that $\Pi_{\mathcal{A}}(X)$ can be expressed as follows:

$$
\begin{aligned}
& \Pi_{\mathcal{A}}(X)=\sum_{\mathcal{Y} \subseteq \mathcal{A} \backslash\{X\}} \frac{1}{1+|\mathcal{Y}|} P((\forall Z \in \mathcal{Y})(\forall W \in \mathcal{A} \backslash(\{X\} \cup \mathcal{Y}))(X=Z>Y)) \\
& =\sum_{\{Y\} \subseteq \mathcal{Y} \subseteq \mathcal{A} \backslash\{X\}} \frac{1}{1+|\mathcal{Y}|} P((\forall Z \in \mathcal{Y})(\forall W \in \mathcal{A} \backslash(\{X\} \cup \mathcal{Y}))(X=Z>W)) \\
& +\sum_{\{Y\} \subseteq \mathcal{Y} \subseteq \mathcal{A} \backslash\{X\}} \frac{1}{1+|\mathcal{Y}|} P((\forall Z \in \mathcal{Y})(\forall W \in \mathcal{A} \backslash(\{X\} \cup \mathcal{Y}))(X=Z>W)) .
\end{aligned}
$$

Recall also that $Q(X, Y)=P(X>Y)+\frac{1}{2} P(X=Y)$. It holds that:

$$
\{X>Y\} \supseteq \bigcup_{\{Y\} \subset \mathcal{Y} \subseteq \mathcal{A} \backslash\{X\}}\{(\forall Z \in \mathcal{Y})(\forall W \in \mathcal{A} \backslash(\{X\} \cup \mathcal{Y}))(X=Z>W)\} .
$$

Moreover, the sets involved are pairwise disjoint, and, consequently,

$$
P(X>Y) \geq \sum_{\{Y\} \not \mathcal{Y} \subseteq \mathcal{A} \backslash\{X\}} P((\forall Z \in \mathcal{Y})(\forall W \in \mathcal{A} \backslash(\{X\} \cup \mathcal{Y}))(X=Z>W)) .
$$

Similarly, it holds that

$$
\{X=Y\} \supseteq \bigcup_{\{Y\} \subseteq \mathcal{Y} \subseteq \mathcal{A} \backslash\{X\}}\{(\forall Z \in \mathcal{Y})(\forall W \in \mathcal{A} \backslash(\{X\} \cup \mathcal{Y}))(X=Z>W)\} .
$$

Since these sets are pairwise disjoint, it holds that:

$$
P(X=Y) \geq \sum_{\{Y\} \subseteq \mathcal{Y} \subseteq \mathcal{A} \backslash\{X\}} P((\forall Z \in \mathcal{Y})(\forall W \in \mathcal{A} \backslash(\{X\} \cup \mathcal{Y}))(X=Z>W))
$$


Consequently, we obtain:

$$
\begin{aligned}
Q(X, Y)=P(X>Y)+\frac{1}{2} P(X=Y) \\
\geq \sum_{\{Y\} \subseteq \mathcal{Y} \subseteq \mathcal{A} \backslash\{X\}} P((\forall Z \in \mathcal{Y})(\forall W \in \mathcal{A} \backslash(\{X\} \cup \mathcal{Y}))(X=Z>W)) \\
\quad+\frac{1}{2} \sum_{\{Y\} \subseteq \mathcal{Y} \subseteq \mathcal{A} \backslash\{X\}} P((\forall Z \in \mathcal{Y})(\forall W \in \mathcal{A} \backslash(\{X\} \cup \mathcal{Y}))(X=Z>W)) \\
\geq \sum_{\{Y\} \subseteq \mathcal{Y} \subseteq \mathcal{A} \backslash\{X\}} \frac{1}{1+|\mathcal{Y}|} P((\forall Z \in \mathcal{Y})(\forall W \in \mathcal{A} \backslash(\{X\} \cup \mathcal{Y}))(X=Z>W)) \\
\quad+\sum_{\{Y\} \subseteq \mathcal{Y} \subseteq \mathcal{A} \backslash\{X\}} \frac{1}{1+|\mathcal{Y}|} P((\forall Z \in \mathcal{Y})(\forall W \in \mathcal{A} \backslash(\{X\} \cup \mathcal{Y}))(X=Z>W)) \\
=\sum_{\mathcal{Y} \subseteq \mathcal{A} \backslash\{X\}} \frac{1}{1+|\mathcal{Y}|} P((\forall Z \in \mathcal{Y})(\forall W \in \mathcal{A} \backslash(\{X\} \cup \mathcal{Y}))(X=Z>W)) \\
=\Pi_{\mathcal{A}}(X) .
\end{aligned}
$$

We conclude that $Q(X, Y) \geq \Pi_{\mathcal{A}}(X)$. Consequently, if $\Pi_{\mathcal{A}}(X) \geq \frac{1}{2}$, then it holds that $X \succeq_{\mathrm{PP}} \mathcal{A} \backslash\{X\}$ and $X \succeq_{\mathrm{SP}} Y$, for any $Y \in \mathcal{A} \backslash\{X\}$.

Next, we establish a connection between pairwise and multivariate winning probabilities. For this aim, we first prove the following technical result.

Lemma 12. Let $\mathcal{A}$ be a finite set of distinct random variables. For any $X \in \mathcal{A}$, it holds that:

$$
\begin{aligned}
& Q\left(X, \max _{Y \in \mathcal{A} \backslash\{X\}} Y\right)-\Pi_{\mathcal{A}}(X)= \\
& \sum_{\emptyset \neq \mathcal{Y} \subseteq \mathcal{A} \backslash\{X\}}\left(\frac{1}{2}-\frac{1}{1+|\mathcal{Y}|}\right) P((\forall Z \in \mathcal{Y})(\forall W \in \mathcal{A} \backslash(\{X\} \cup \mathcal{Y}))(X=Z>W)) .
\end{aligned}
$$

Proof. Consider the expression of $Q\left(X, \max _{Y \in \mathcal{A} \backslash\{X\}} Y\right)$ :

$$
\begin{aligned}
Q(X, & \left.\max _{Y \in \mathcal{A} \backslash\{X\}} Y\right)=P\left(X>\max _{Y \in \mathcal{A} \backslash\{X\}} Y\right) \\
& +\frac{1}{2} \sum_{\emptyset \neq \mathcal{Y} \subseteq \mathcal{A} \backslash\{X\}} P((\forall Z \in \mathcal{Y})(\forall W \in \mathcal{A} \backslash(\{X\} \cup \mathcal{Y}))(X=Z>W)) .
\end{aligned}
$$


Using Eq. [5], $\Pi_{\mathcal{A}}(X)$ can be expressed as:

$$
\begin{aligned}
& \Pi_{\mathcal{A}}(X)=P\left(X>\max _{Y \in \mathcal{A} \backslash\{X\}} Y\right)+ \\
& +\sum_{\emptyset \neq \mathcal{Y} \subseteq \mathcal{A} \backslash\{X\}} \frac{1}{1+|\mathcal{Y}|} P((\forall Z \in \mathcal{Y})(\forall W \in \mathcal{A} \backslash(\{X\} \cup \mathcal{Y}))(X=Z>W)) .
\end{aligned}
$$

The stated result follows easily by computing the difference between both expressions.

From the preceding result, we deduce that

$$
\Pi_{\mathcal{A}}(X) \leq Q\left(X, \max _{Y \in \mathcal{A} \backslash\{X\}} Y\right) .
$$

Clearly, if $X \succeq_{\mathrm{PP}} \mathcal{A} \backslash\{X\}$ with multivariate winning probability $\Pi_{\mathcal{A}}(X) \geq \frac{1}{2}$, then Eq. [6] implies that $X \succeq_{\mathrm{SP}} \max _{Y \in \mathcal{A} \backslash\{X\}} Y$.

Moreover, there are situations where the inequality in Eq. (7) becomes an equality.

Proposition 13. Let $\mathcal{A}$ be a finite set of distinct random variables and consider $X \in \mathcal{A}$. It holds that

$$
\Pi_{\mathcal{A}}(X)=Q\left(X, \max _{Y \in \mathcal{A} \backslash\{X\}} Y\right)
$$

if and only if for every $\mathcal{Y} \subseteq \mathcal{A} \backslash\{X\}$ with $|\mathcal{Y}|>1$ :

$$
P((\forall Z \in \mathcal{Y})(\forall W \in \mathcal{A} \backslash(\{X\} \cup \mathcal{Y}))(X=Z>W))=0 .
$$

Proof. From Lemma 12, $Q\left(X, \max _{Y \in \mathcal{A} \backslash\{X\}} Y\right)-\Pi_{\mathcal{A}}(X)$ is the sum of non-negative terms, hence $Q\left(X, \max _{Y \in \mathcal{A} \backslash\{X\}} Y\right)=\Pi_{\mathcal{A}}(X)$ if and only if all the terms are zero, which is equivalent to

$$
\left(\frac{1}{2}-\frac{1}{1+|\mathcal{Y}|}\right) P((\forall Z \in \mathcal{Y})(\forall W \in \mathcal{A} \backslash(\{X\} \cup \mathcal{Y}))(X=Z>W))=0
$$

for every $\emptyset \neq \mathcal{Y} \subseteq \mathcal{A} \backslash\{X\}$. However, for $\mathcal{Y} \subseteq \mathcal{A} \backslash\{X\}$ of cardinality 1, Eq. (10) trivially holds because $\frac{1}{2}-\frac{1}{1+|\mathcal{Y}|}=0$. Hence, Eq. (8) holds if and only if for every $\mathcal{Y} \subseteq \mathcal{A} \backslash\{X\}$ with $|\mathcal{Y}|>1$, Eq. (9) holds.

In particular, the above result holds when the random variables satisfy $P(X=Y)=0$ for any $Y \in \mathcal{A} \backslash\{X\}$, as is for instance the case for discrete 
random variables with pairwise disjoint supports or when the random variables in $\mathcal{A}$ form an absolutely continuous random vector.

The following example shows that when Eq. (9) is not satisfied, it is in general not possible to express multivariate winning probabilities in terms of the probabilistic relation $Q$.

Example 14. Consider the space $\Omega=\left\{\omega_{1}, \omega_{2}, \omega_{3}, \omega_{4}\right\}$ and the probability measure $P$ given by

$$
P\left(\left\{\omega_{1}\right\}\right)=0.1, \quad P\left(\left\{\omega_{2}\right\}\right)=0.2, \quad P\left(\left\{\omega_{3}\right\}\right)=0.3, \quad P\left(\left\{\omega_{4}\right\}\right)=0.4 .
$$

Consider the set of random variables $\mathcal{A}=\{X, Y, Z\}$ defined by:

\begin{tabular}{c|cccc} 
& $\omega_{1}$ & $\omega_{2}$ & $\omega_{3}$ & $\omega_{4}$ \\
\hline$X$ & 1 & 0 & 1 & 0 \\
$Y$ & 0 & 1 & 1 & 0 \\
$Z$ & 1 & 1 & 0 & 0
\end{tabular}

For these random variables, it holds that:

$$
\Pi_{\mathcal{A}}(X)=\frac{1}{3}, \quad \Pi_{\mathcal{A}}(Y)=\frac{1.15}{3}, \quad \Pi_{\mathcal{A}}(Z)=\frac{0.85}{3},
$$

whence $Y \succ_{\mathrm{PP}} X \succ_{\mathrm{PP}} Z$. Note that

$$
\Pi_{\mathcal{A}}(Y) \neq Q(Y, \max (X, Z))=0.45 .
$$

Not surprisingly, one can easily verify that Eq. (9) is not satisfied for the random variable $Y$.

Finally, let us generalize Theorem 5 and provide a characterization of probabilistic preference. To that end, we consider a set of random variables $\mathcal{A}$ such that every $X \in \mathcal{A}$ satisfies Eq. (9).

Theorem 15. Let $\mathcal{A}$ be a finite set of distinct random variables such that any $X \in \mathcal{A}$ and $\mathcal{Y} \subseteq \mathcal{A} \backslash\{X\}$ with $|\mathcal{Y}|>1$ satisfy Eq. (9). Then $X \succeq_{\mathrm{PP}} \mathcal{A} \backslash\{X\}$ if and only if

$$
F_{X-\max _{Z \in \mathcal{A} \backslash\{X\}} Z}(0) \leq F_{Y-\max _{Z \in \mathcal{A} \backslash\{Y\}} Z}(0),
$$

for any $Y \in \mathcal{A} \backslash\{X\}$. 
Proof. The probabilistic relation $Q(X, Y)$ can be expressed as:

$$
\begin{aligned}
Q(X, Y) & =P(X>Y)+\frac{1}{2} P(X=Y) \\
& =1-P(X \leq Y)+\frac{1}{2} P(X=Y)=1-F_{X-Y}(0)+\frac{1}{2} P(X=Y) .
\end{aligned}
$$

Using this expression with $Y=\max _{Z \in \mathcal{A} \backslash\{X\}} Z$ and applying Proposition 13 , it follows that:

$$
\begin{aligned}
\Pi_{\mathcal{A}}(X) & =Q\left(X \max _{Z \in \mathcal{A} \backslash\{X\}} Z\right) \\
& =1-F_{X-\max _{Z \in \mathcal{A} \backslash\{X\}} Z}(0)+\frac{1}{2} P\left(X=\max _{Z \in \mathcal{A} \backslash\{X\}} Z\right) \\
& =1-F_{X-\max _{Z \in \mathcal{A} \backslash\{X\}} Z}(0) .
\end{aligned}
$$

Similarly, we can compute $\Pi_{\mathcal{A}}(Y)$ :

$$
\Pi_{\mathcal{A}}(Y)=1-F_{Y-\max _{Z \in \mathcal{A} \backslash\{Y\}} Z}(0) .
$$

Therefore, $X \succeq_{\mathrm{PP}} Y$ in $\mathcal{A}$ if and only if:

$$
1-F_{X-\max _{Z \in \mathcal{A} \backslash\{X\}} Z}(0) \geq 1-F_{Y-\max _{Z \in \mathcal{A} \backslash\{Y\}} Z}(0),
$$

or, equivalently,

$$
F_{X-\max _{Z \in \mathcal{A} \backslash\{X\}} Z}(0) \leq F_{Y-\max _{Z \in \mathcal{A} \backslash\{Y\}} Z}(0) .
$$

In particular, $X \succeq_{\mathrm{PP}} \mathcal{A} \backslash\{X\}$ if the above inequality holds for every $Y \in \mathcal{A} \backslash\{X\}$.

Thus, given a set of random variables $\mathcal{A}$ satisfying the conditions of Theorem 15. finding the preferred one by computing multivariate winning probabilities is equivalent to comparing the values of $F_{\left.X-\max _{Z \in \mathcal{A} \backslash\{X\}} Z\right)}(0)$ for every $X \in \mathcal{A}$.

\section{Stochastic dominance versus probabilistic preference}

Although stochastic dominance and statistical preference have different interpretations [11, there are situations in which they are closely connected. 
Proposition 16. [11, 13, 22] If $X$ and $Y$ are two independent random variables such that $X \succeq_{\mathrm{FSD}} Y$, then it also holds that $X \succeq_{\mathrm{SP}} Y$.

This result underlines that for two independent random variables, stochastic dominance is more restrictive than statistical preference. Also in the multivariate setting, we can establish an interesting correspondence between stochastic dominance and probabilistic preference when the random variables are independent. This lack of dependence clearly compensates for the fact that stochastic dominance only considers the marginal distributions.

Theorem 17. Let $\mathcal{A}$ be a finite set of distinct random variables such that any $X \in \mathcal{A}$ and $\mathcal{Y} \subseteq \mathcal{A} \backslash\{X\}$ with $|\mathcal{Y}|>1$ satisfy Eq. (9). Assume that the random variables in $\mathcal{A}$ are independen $\biguplus^{3}$. If $X \succeq_{\text {FSD }} Y$ for any $Y \in \mathcal{A} \backslash\{X\}$, then $X \succeq_{\mathrm{PP}} \mathcal{A} \backslash\{X\}$.

Proof. Since the conditions of Proposition 13 hold, we have that:

$$
\begin{aligned}
& \Pi_{\mathcal{A}}(X)=Q\left(X, \max _{Z \in \mathcal{A} \backslash\{X\}} Z\right) \text { and } \\
& \Pi_{\mathcal{A}}(Y)=Q\left(Y, \max _{Z \in \mathcal{A} \backslash\{Y\}} Z\right),
\end{aligned}
$$

for any $Y \in \mathcal{A} \backslash\{X\}$. Therefore, $X \succeq_{\mathrm{PP}} \mathcal{A} \backslash\{X\}$ if and only if:

$$
Q\left(X, \max _{Z \in \mathcal{A} \backslash\{X\}} Z\right) \geq Q\left(Y, \max _{Z \in \mathcal{A} \backslash\{Y\}} Z\right)
$$

for any $Y \in \mathcal{A} \backslash\{X\}$. Note that since the random variables in $\mathcal{A}$ are independent, we also have that:

1. $X$ and $\max _{Z \in \mathcal{A} \backslash\{X\}} Z$ are independent;

2. $Y$ and $\max _{Z \in \mathcal{A} \backslash\{Y\}} Z$ are independent.

Now, we have to recall that if $U_{1}$ and $U_{2}$ are two independent random variables with cumulative distribution functions $F_{U_{1}}$ and $F_{U_{2}}$, as a consequence of [29], Thm. 20.3], it holds that $P\left(U_{1} \geq U_{2}\right)=E\left[F_{U_{2}}\left(U_{1}\right)\right]$.

\footnotetext{
${ }^{3}$ Note that in some contexts, independence is also referred to as mutual independence to distinguish it from pairwise independence.
} 
Applying this result, we have that:

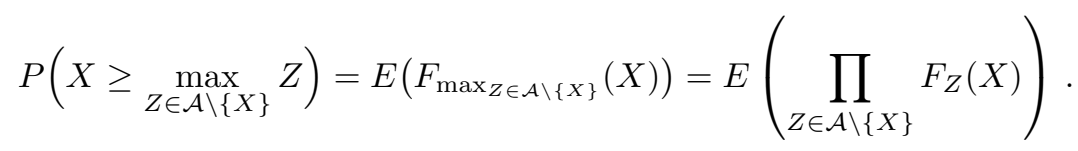

Similarly,

$$
\begin{aligned}
P\left(Y \geq \max _{Z \in \mathcal{A} \backslash\{Y\}} Z\right) & =E\left(F_{\left.\max _{Z \in \mathcal{A} \backslash\{Y\}}(Y)\right)}\right. \\
& =E\left(\prod_{Z \in \mathcal{A} \backslash\{Y\}} F_{Z}(Y)\right) \leq E\left(\prod_{Z \in \mathcal{A} \backslash\{X\}} F_{Z}(Y)\right),
\end{aligned}
$$

where the last inequality holds since $X \succeq_{\mathrm{FSD}} X_{i}$. Finally, Eq. (1) assures that $E[h(X)] \geq E\left[h\left(X_{i}\right)\right]$ for any increasing function $h$. In particular, we may consider the increasing function

$$
h(t)=\prod_{j=1}^{n} F_{X_{j}}(t) .
$$

Therefore, for any $Y \in \mathcal{A} \backslash\{X\}$, it holds that

$$
\begin{aligned}
P\left(X \geq \max _{Z \in \mathcal{A} \backslash\{X\}} Z\right) & =E\left(\prod_{Z \in \mathcal{A} \backslash\{X\}} F_{Z}(X)\right) \\
& \geq E\left(\prod_{Z \in \mathcal{A} \backslash\{X\}} F_{Z}(Y)\right) \geq P\left(Y \geq \max _{Z \in \mathcal{A} \backslash\{Y\}} Z\right),
\end{aligned}
$$

or, equivalently,

$$
Q\left(X, \max _{Z \in \mathcal{A} \backslash\{X\}} Z\right) \geq Q\left(Y, \max _{Z \in \mathcal{A} \backslash\{Y\}} Z\right)
$$

We conclude that $X \succeq_{\mathrm{PP}} Y$ for every $Y \in \mathcal{A} \backslash\{X\}$, which implies that $X \succeq_{\mathrm{PP}}$ $\mathcal{A} \backslash\{X\}$.

\section{Probabilistic preference as a tool for linguistic decision making}

We have introduced probabilistic preference as a tool for the comparison of two or more random variables. As an illustration of the usefulness of this tool, we consider a decision making problem with linguistic utilities. We consider 
the product management example discussed in [30, Section 8]: a company seeks to select its production strategy for the next year, and considers six possible alternatives:

$A_{1}$ Create a new product for very high-income customers.

$A_{2}$ Create a new product for high-income customers.

$A_{3}$ Create a new product for medium-income customers.

$A_{4}$ Create a new product for low-income customers.

$A_{5}$ Create a new product suitable for all customers.

$A_{6}$ Do not create a new product.

Due to the uncertainty involved, the experts of the company are not able to express the impact of each alternative in a numerical way. For this reason, they express the utility on a seven-point linguistic scale $S=\left\{s_{1}, \ldots, s_{7}\right\}$, where:

$$
\begin{array}{lll}
s_{1}: \text { None } & s_{2}: \text { Very low } & s_{3}: \text { Low } \\
s_{4}: \text { Medium } & s_{5}: \text { High } & s_{6}: \text { Very high } \\
s_{7}: \text { Perfect } & &
\end{array}
$$

Moreover, since the decision depends on the economic situation of the following year, six scenarios are considered:

$$
\begin{array}{lll}
N_{1}: \text { Very bad } & N_{2}: \text { Bad } & N_{3}: \text { Regular-Bad } \\
N_{4}: \text { Regular-Good } & N_{5}: \text { Good } & N_{6}: \text { Very good }
\end{array}
$$

The following probability vector is given for these scenarios:

$$
W=(0.1,0.1,0.1,0.2,0.2,0.3) .
$$

The first expert $e_{1}$ of the company gives the following evaluations of the alternatives:

\begin{tabular}{lllllll}
\hline$e_{1}$ & $N_{1}$ & $N_{2}$ & $N_{3}$ & $N_{4}$ & $N_{5}$ & $N_{6}$ \\
\hline$A_{1}$ & $s_{2}$ & $s_{1}$ & $s_{4}$ & $s_{6}$ & $s_{7}$ & $s_{5}$ \\
$A_{2}$ & $s_{1}$ & $s_{3}$ & $s_{5}$ & $s_{5}$ & $s_{6}$ & $s_{6}$ \\
$A_{3}$ & $s_{3}$ & $s_{4}$ & $s_{4}$ & $s_{4}$ & $s_{4}$ & $s_{7}$ \\
$A_{4}$ & $s_{2}$ & $s_{5}$ & $s_{6}$ & $s_{4}$ & $s_{2}$ & $s_{5}$ \\
$A_{5}$ & $s_{1}$ & $s_{3}$ & $s_{4}$ & $s_{5}$ & $s_{6}$ & $s_{6}$ \\
$A_{6}$ & $s_{6}$ & $s_{5}$ & $s_{5}$ & $s_{4}$ & $s_{2}$ & $s_{2}$ \\
\hline
\end{tabular}


Let us denote by $\mathcal{A}$ the set of alternatives to be compared. Computing the multivariate winning probabilities of $e_{1}$ for each alternative, we obtain the following:

$$
\begin{array}{c|cccccc}
A_{i} & A_{1} & A_{2} & A_{3} & A_{4} & A_{5} & A_{6} \\
\hline \Pi_{\mathcal{A}}^{e_{1}}\left(A_{i}\right) & 0.4 & 0 & 0.3 & 0.15 & 0 & 0.15
\end{array}
$$

Note that for computing the multivariate winning probabilities $\Pi_{\mathcal{A}}^{e_{1}}\left(A_{i}\right)$, we have made use of Proposition 10. Since for the first expert it holds that $A_{2}, A_{5}<$ $\max \left(A_{1}, A_{3}, A_{4}, A_{6}\right)$, we know that the multivariate winning probability of $A_{2}$ and $A_{5}$ for the first expert is zero; hence, we can simplify the computations and simply use the formula for $\Pi_{\mathcal{A}^{\prime}}$, where $\mathcal{A}^{\prime}=\left\{A_{1}, A_{3}, A_{4}, A_{6}\right\}$. The preferences of expert $e_{1}$ are then given by: $A_{1} \succ_{\mathrm{PP}} A_{3} \succ_{\mathrm{PP}} A_{4} \equiv_{\mathrm{PP}} A_{6} \succ_{\mathrm{PP}} A_{2} \succ_{\mathrm{PP}} A_{5}$.

Two other experts of the company, $e_{2}$ and $e_{3}$, also evaluate the alternatives:

\begin{tabular}{lllllll}
\hline$e_{2}$ & $N_{1}$ & $N_{2}$ & $N_{3}$ & $N_{4}$ & $N_{5}$ & $N_{6}$ \\
\hline$A_{1}$ & $s_{3}$ & $s_{1}$ & $s_{3}$ & $s_{5}$ & $s_{6}$ & $s_{6}$ \\
$A_{2}$ & $s_{1}$ & $s_{3}$ & $s_{4}$ & $s_{5}$ & $s_{6}$ & $s_{6}$ \\
$A_{3}$ & $s_{3}$ & $s_{4}$ & $s_{5}$ & $s_{4}$ & $s_{3}$ & $s_{7}$ \\
$A_{4}$ & $s_{3}$ & $s_{4}$ & $s_{5}$ & $s_{4}$ & $s_{2}$ & $s_{4}$ \\
$A_{5}$ & $s_{2}$ & $s_{3}$ & $s_{4}$ & $s_{6}$ & $s_{6}$ & $s_{6}$ \\
$A_{6}$ & $s_{7}$ & $s_{6}$ & $s_{4}$ & $s_{3}$ & $s_{2}$ & $s_{2}$ \\
\hline
\end{tabular}

\begin{tabular}{lllllll}
\hline$e_{3}$ & $N_{1}$ & $N_{2}$ & $N_{3}$ & $N_{4}$ & $N_{5}$ & $N_{6}$ \\
\hline$A_{1}$ & $s_{1}$ & $s_{2}$ & $s_{3}$ & $s_{5}$ & $s_{7}$ & $s_{6}$ \\
$A_{2}$ & $s_{2}$ & $s_{3}$ & $s_{4}$ & $s_{4}$ & $s_{5}$ & $s_{6}$ \\
$A_{3}$ & $s_{3}$ & $s_{4}$ & $s_{6}$ & $s_{4}$ & $s_{3}$ & $s_{7}$ \\
$A_{4}$ & $s_{2}$ & $s_{4}$ & $s_{6}$ & $s_{4}$ & $s_{2}$ & $s_{4}$ \\
$A_{5}$ & $s_{1}$ & $s_{3}$ & $s_{4}$ & $s_{5}$ & $s_{6}$ & $s_{6}$ \\
$A_{6}$ & $s_{6}$ & $s_{6}$ & $s_{5}$ & $s_{3}$ & $s_{2}$ & $s_{3}$ \\
\hline
\end{tabular}

Following a similar procedure, we can compute the multivariate winning probabilities for experts $e_{2}$ and $e_{3}$ :

\begin{tabular}{c|cccccc}
$A_{i}$ & $A_{1}$ & $A_{2}$ & $A_{3}$ & $A_{4}$ & $A_{5}$ & $A_{6}$ \\
\hline$\Pi_{\mathcal{A}}^{e_{2}}\left(A_{i}\right)$ & 0.0667 & 0.0667 & 0.35 & 0.05 & 0.2667 & 0.2 \\
$\Pi_{\mathcal{A}}^{e_{3}}\left(A_{i}\right)$ & 0.3 & 0 & 0.35 & 0.05 & 0.1 & 0.2
\end{tabular}

For the company, the three experts do not have the same influence, and their importance is given by the weight vector $(0.2,0.4,0.4)$. Hence, using the multivariate winning probabilities $\Pi_{\mathcal{A}}^{e_{j}}\left(A_{i}\right)$ and the weight vector of the experts $(0.2,0.4,0.4)$, we can aggregate them to obtain the overall multivariate winning probability of each alternative:

$$
\begin{aligned}
\Pi_{\mathcal{A}}\left(A_{1}\right) & =\Pi_{\mathcal{A}}^{e_{1}}\left(A_{1}\right) \cdot 0.2+\Pi_{\mathcal{A}}^{e_{2}}\left(A_{1}\right) \cdot 0.4+\Pi_{\mathcal{A}}^{e_{3}}\left(A_{1}\right) \cdot 0.4 \\
& =0.4 \cdot 0.2+0.0667 \cdot 0.4+0.3 \cdot 0.4=0.22667 .
\end{aligned}
$$


Similarly, for the other alternatives we obtain:

\begin{tabular}{c|cccccc}
$A_{i}$ & $A_{1}$ & $A_{2}$ & $A_{3}$ & $A_{4}$ & $A_{5}$ & $A_{6}$ \\
\hline$\Pi_{\mathcal{A}}\left(A_{i}\right)$ & 0.22667 & 0.02667 & 0.34 & 0.07 & 0.14667 & 0.19
\end{tabular}

Thus, the multivariate winning probabilities yield $A_{3}$ as the preferred alternative: $A_{3} \succ_{\mathrm{PP}} \mathcal{A} \backslash\left\{A_{3}\right\} ; A_{1}$ is the second preferred alternative, $A_{6}$ the third, $A_{5}$ the fourth, $A_{4}$ the fifth and finally $A_{2}$ is the least preferred alternative. Consequently, creating a new product for medium-income customers seems to be the best option, while the worst alternative is creating a new product for highincome customers. Hence, the final ranking given by probabilistic preference relation is $A_{3} \succ_{\mathrm{PP}} A_{1} \succ_{\mathrm{PP}} A_{6} \succ_{\mathrm{PP}} A_{5} \succ_{\mathrm{PP}} A_{4} \succ_{\mathrm{PP}} A_{2}$.

This problem was solved in [30] by means of aggregation operators. The main drawback of that approach is that it depends on the chosen aggregation operator. For example, for the maximum operator the final ranking is $A_{3} \succ A_{1} \succ A_{6} \succ$ $A_{2} \sim A_{5} \succ A_{4}$, while for the minimum operator the final ranking is $A_{3} \succ A_{4} \sim$ $A_{6} \succ A_{1} \sim A_{2} \sim A_{5}$. It seems that for most of the aggregation operators the preferred alternative is $A_{3}$, which coincides with the best alternative given by our approach. However, the ranking between the remaining alternatives varies a lot with the aggregation operator.

\section{Conclusions}

In this paper, we have introduced a new method for the comparison of two or more random variables. The proposed method, called probabilistic preference, is based on multivariate winning probabilities, which express the strength of preference of one random variable over the other ones in a given set. Since multivariate winning probabilities are based on the joint distribution of the random variables, probabilistic preference takes into account the stochastic dependences between them. Also, multivariate winning probabilities establish a ranking (with ties) on the set of random variables.

We have also investigated some properties of the notion of probabilistic preference, and provided a characterization in terms of the pointwise comparison of 
some specific cumulative distribution functions. In addition, we have confirmed that there is no relationship between probabilistic preference and statistical preference, simply because the latter only takes into account the bivariate distributions. With respect to stochastic dominance, we have shown the interesting result that for independent random variables, first degree stochastic dominance implies probabilistic preference.

Note that this new method could offer an interesting alternative in some realworld applications in which both stochastic dominance and statistical preference do not provide an optimal solution, as, for example, in multicriteria decision making.

As for future research, we have stressed that one of the advantages of using multivariate winning probabilities is that they use the joint distribution of the random variables and thus take into account the possible dependence between them. At this point, we can make use of the Theory of Copulas [31, which allows us to capture the dependence information between the random variables. Therefore, as we did in [14 for statistical preference, we aim to extend the connection between first degree stochastic dominance and probabilistic preference given in Theorem 17 for some prominent dependence models.

Also, it would be interesting to study the behaviour of probabilistic preference for some well-known families of distributions, such as normally distributed random variables.

Finally, the mapping $\Pi_{\mathcal{A}}$ could be studied within the framework of probabilistic and fuzzy choice functions (see [32] for an overview and [33, 34] for some recent advances), determining which properties it satisfies, as well as from the point of view of game theory.

\section{Acknowledgements}

The research reported in this paper has been supported by Project TIN201459543-P. We want to thank the Area Editor as well as the anonymous reviewers for their insightful comments that helped us to improve the paper. 


\section{References}

[1] H. Levy, Stochastic Dominance, Kluwer Academic Publishers, 1998.

[2] A. Müller, D. Stoyan, Comparison Methods for Stochastic Models and Risks, Wiley, 2002.

[3] M. Shaked, J. G. Shanthikumar, Stochastic Orders, Springer-Verlag, New York, 2007.

[4] B. De Schuymer, H. De Meyer, B. De Baets, A fuzzy approach to stochastic dominance of random variables, Lecture Notes in Artificial Intelligence 2715, 2003, pp. 253-260.

[5] B. De Schuymer, H. De Meyer, B. De Baets, S. Jenei, On the cycletransitivity of the dice model, Theory and Decision 54 (2003) 261-285.

[6] M. Islam, J. Braden, Bio-economics development of floodplains: Farming versus fishing in Bangladesh, Environment and Development Economics 11(1) (2006) 95-126.

[7] N. Noyan, G. Rudolf, A. Ruszczyński, Relaxations of linear programming problems with first order stochastic dominance constraints, Operations Research L 34 (2006) 653-659.

[8] M. A. Arcones, P. H. Kvam, F. J. Samaniego, Nonparametric estimation of a distribution subject to a stochastic preference constraint, Journal of the American Statistical Association 97 (2002) 170-182.

[9] X. Li, X. Hu, Some new stochastic comparisons for redundancy allocations in series and parallel systems, Statistics and Probability Letters 78 (2008) 3388-3394.

[10] C. Wrather, P. L. Yu, Probability dominance in random outcomes, Journal of Optimization Theory and Applications 36 (1982) 315-334. 
[11] I. Montes, D. Martinetti, S. Díaz, S. Montes, Interpretation of statistical preference in terms of location parameters, INFOR: Information Systems and Operational Research 53 (2015) 1-12.

[12] I. Montes, D. Martinetti, S. Díaz, S. Montes, Comparison of random variables coupled by Archimedean copulas, in: C. Borgelt, G. GonzálezRodríguez, W. Trutschnig, M. Lubiano, M. Gil, P. Grzegorzewski, O. Hryniewicz (Eds.), Combining Soft Computing and Statistical Methods in Data Analysis, volume 77 of Advances in Intelligent and Soft Computing, Springer Berlin / Heidelberg, 2010, pp. 467-474.

[13] I. Montes, D. Martinetti, S. Díaz, S. Montes, Statistical preference as a tool in consensus processes, in: E. Herrera-Viedma, J. García-Lapresta, J. Kacprzyk, M. Fedrizzi, H. Nurmi, S. Zadrozny (Eds.), Consensual Processes, volume 267 of Studies in Fuzziness and Soft Computing, Springer Berlin / Heidelberg, 2011, pp. 65-92.

[14] I. Montes, S. Montes, Stochastic dominance and statistical preference for random variables coupled by an Archimedean copula or by the FréchetHoeffding upper bound, Journal of Multivariate Analysis 143 (2016) 275298.

[15] B. De Schuymer, H. De Meyer, B. De Baets, Cycle-transitive comparison of independent random variables, Journal of Multivariate Analysis 96 (2005) $352-373$.

[16] I. Montes, D. Martinetti, S. Díaz, S. Montes, Estudio de la preferencia estadística en distribuciones normales bidimensionales, in: Proceedings of XXXII SEIO Conference, 2010, p. 78.

[17] J. von Neumann, O. Morgenstern, Theory of Games and Economic Behavior, Princeton University Press, 1953.

[18] J. Durba, F. Maccheroni, E. Ok, Expected utility theory without the completeness axiom, Journal of Economic Theory 115 (2004) 118-133. 
[19] M. Condorcet, An essay on the application of probability theory to plurality decision making: an election between three candidates, Reprinted in 1989, F. Sommerlad, I. McLean (Eds.), 1785.

[20] D. Dubois, H. Fargier, P. Perny, Qualitative decision theory with preference relations and comparative uncertainty: An axiomatic approach, Artificial Intelligence 148 (2003) 219-260.

[21] B. De Baets, K. De Loof, H. De Meyer, A frequentist view on cycletransitivity of reciprocal relations, Fuzzy Sets and Systems 281 (2015) 198-218.

[22] B. De Baets, H. De Meyer, Transitivity frameworks for reciprocal relations: cycle-transitivity versus FG-transitivity, Fuzzy Sets and Systems 152 (2005) 249-270.

[23] B. De Baets, H. De Meyer, On the cycle-transitive comparison of artificially coupled random variables, International Journal of Approximate Reasoning 47 (2008) 306-322.

[24] B. De Baets, H. De Meyer, K. De Loof, On the cycle-transitivity of the mutual rank probability relation of a poset, Fuzzy Sets and Systems 161 (2010) 2695-2708.

[25] B. De Baets, H. De Meyer, B. De Schuymer, S. Jenei, Cyclic evaluation of transitivity of reciprocal relations, Social Choice and Welfare 26 (2006) $217-238$.

[26] H. De Meyer, B. De Baets, B. De Schuymer, On the transitivity of the comonotonic and countermonotonic comparison of random variables, Journal of Multivariate Analysis 98 (2007) 177-193.

[27] B. De Schuymer, H. De Meyer, B. De Baets, Extreme copulas and the comparison of ordered lists, Theory and Decision 62 (2007) 195-217. 
[28] D. Martinetti, I. Montes, S. Díaz, S. Montes, A study on the transitivity of probabilistic and fuzzy relations, Fuzzy Sets and Systems 184 (2011) $156-170$.

[29] P. Billingsley, Probability and Measure, 3 ed., Wiley-Interscience, 1995.

[30] J. M. Merigó, A. M. Gil-Lafuente, Induced 2-tuple linguistic generalized aggregation operators and their application in decision-making, Information Sciences 236 (2013) 1-16.

[31] R. B. Nelsen, An Introduction to Copulas, Springer, 1998.

[32] X. Wang, C. Wu, X. Wu, Choice functions in fuzzy environment: An overview, Studies in Fuzziness and Soft Computing 261 (2010) 149-169.

[33] D. Martinetti, S. Montes, S. Díaz, B. De Baets, On a correspondence between probabilistic and fuzzy choice functions, Fuzzy Optimization and Decision Making 17 (2018) 247-264.

[34] D. Martinetti, S. Montes, S. Díaz, B. De Baets, On Arrow-Sen style equivalences between rationality conditions for fuzzy choice functions, Fuzzy Optimization and Decision Making 13 (2014) 369-396. 